Document downloaded from:

http://hdl.handle.net/10251/101802

This paper must be cited as:

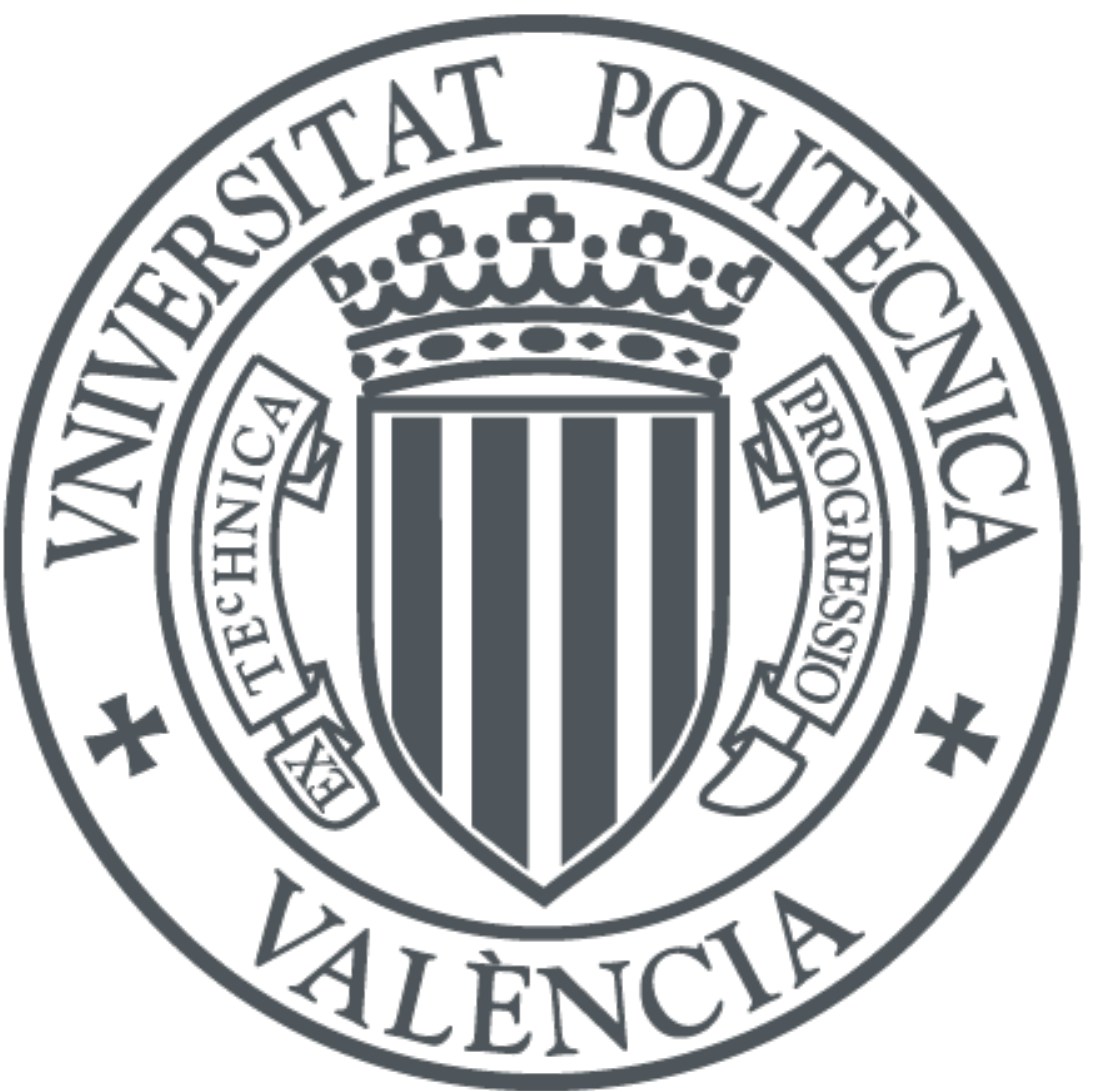

The final publication is available at

https://doi.org/10.1007/978-3-319-65151-4_4

Copyright Springer

Additional Information 


\title{
A Holistic Algorithm for Materials Requirement Planning in Collaborative Networks
}

\author{
Beatriz Andres, Raul Poler and Raquel Sanchis \\ Research Centre on Production Management and Engineering (CIGIP). Universitat \\ Politècnica de València (UPV). Calle Alarcón, 03801 Alcoy, Spain \\ \{bandres, rpoler, rsanchis\}@cigip.upv.es
}

\begin{abstract}
Collaboration has increasingly been considered a key topic within the small and medium-sized enterprises, allowing dealing with the intense competitiveness of today's globalised markets. The European H2020 Cloud Collaborative Manufacturing Networks Project proposes mechanisms to encourage collaboration among enterprises, through the computation of collaborative plans. Particularly, this paper focuses on the proposal of a holistic algorithm to deal with the automated and collaborative calculation of the Materials Requirement Plan. The proposed algorithm is validated in a collaborative network belonging to the automotive industry.
\end{abstract}

Keywords: materials requirement plan, collaboration, cloud computing, data exchange, automotive sector, collaborative planning, Cloud Collaborative Manufacturing Networks, H2020 Project.

\section{Introduction}

Collaboration has increasingly been considered a key topic within the small and medium-sized (SME) enterprises (SMEs), allowing dealing with the intense competitiveness of today's globalised markets. The SMEs participation in collaborative networks increases their competitive advantages over large multinational corporations that produce on a large scale, and mass customised products. This situation has involved the design of novel models, algorithms, mechanisms and tools to support companies on establishing collaborative relationships with the network partners. Nevertheless, SMEs are characterised by the scarce of resources to access such collaborative tools. In addition a cultural change is necessary within the SMEs, in the context of exchanging information and calculating their plans collaboratively, with the main aim of obtaining more realistic plans that are beneficial to all partners in the network.

The European Cloud Collaborative Manufacturing Networks project (C2NET), one of the projects funded under the programme H2020 Technologies for Factories of the Future [1] [2], aims to build a novel Cloud Architecture to provide SMEs affordable tools (in term of cost and usability) to help them to overcome the barriers appearing when they are willing to participate in a collaborative network $(\mathrm{CN})$. C2NET Project generates a Cloud Architecture composed by [3] (see Figure 1): (i) The Data Collection Framework (C2NET DCF) for IoT-based continuous data collection from 
supply network resources; (ii) The Optimiser (C2NET OPT) to support manufacturing networks in the optimisation of manufacturing and logistics assets by the collaborative computation of production, replenishment and delivery plans, to achieve shorter delivery times, higher speed and consistency of schedules; (iii) The Collaboration Tools (C2NET COT) for providing a set of tools in charge of managing the agility of the collaborative processes; and (iv) The Cloud Platform (C2NET CPL) to integrate the data module, the optimisers and the collaborative tools in the cloud and allow the access to process optimisation resources to all the participants in the value chain to support their decisions and process enhancement.

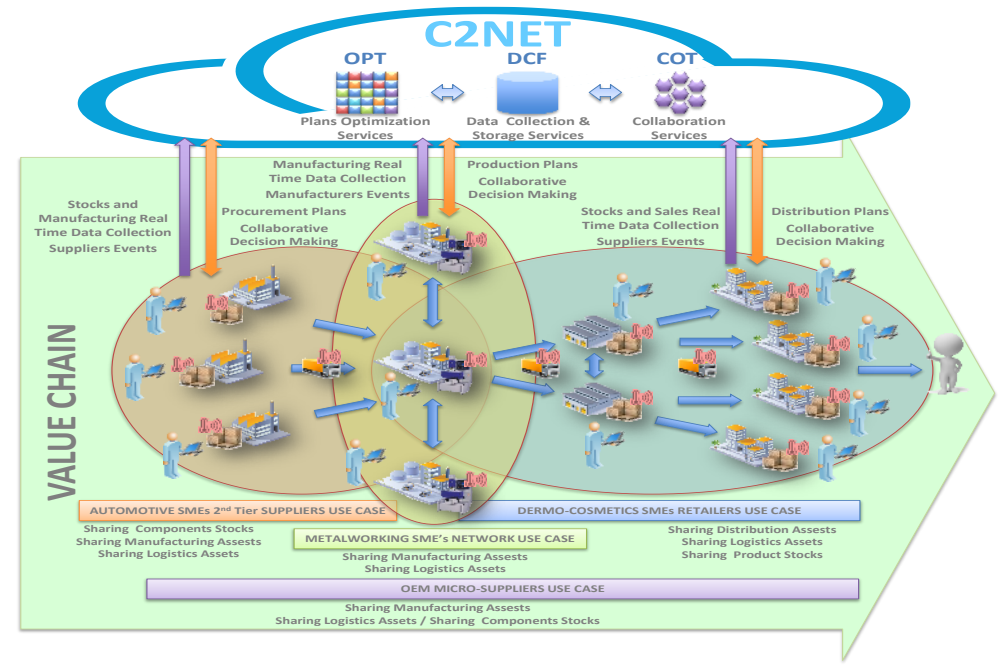

Fig. 1. C2NET Project Results [4]

This paper presents part of the results obtained in the C2NET OPT and C2NET COT used to compute optimised collaborative plans in an automotive network. Particularly, this paper focuses on the proposal of an approach to deal with the automated and collaborative calculation of the Materials Requirement Plan (MRP) of a CN. The complete approach consists of (i) MRP algorithms embedded in the algorithms repository of C2NET OPT, (ii) collaborative mechanisms defined through workflows in the C2NET COT Orchestration Planning Process (OPP) module, and (iii) cloud-supported tool to exchange the data between the collaborative enterprises. In the light of this, the paper is organised as follows: Section 2, in which the problem is identified; in Section 3, the complete approach to develop the present contribution is described; in Section 4, the results of the related experiments are presented; and finally, in Section 5 a discussion of potential generalisation and reuse of the presented results is provided.

\section{Problem Description}

Current planning processes are characterised by uncertainties derived from the continuously and rapidly changing market conditions and increasingly shorter time- 
to-market requirements. The significance of establishing collaborative processes to enhance the SMEs competitiveness and increase their agility and adaptability to deal with rapid evolutions of existing and future markets, is cross-examined and widely studied in [5] [6].

When starting to collaborate, the enterprises find that the replenishment, manufacturing and delivery plans are treated and computed short-sighted, within the enterprise, and no planning results are exchanged with upstream and downstream network partners. In this regard, the enterprises are dependent on complex information exchanges and material flows, requiring new approaches inside and outside the enterprise to support them on the establishment of collaborative planning process.

The problem treated in this paper focuses on the collaborative MRP from the automotive industry, applied in a network in which first and second tiers participate. In the current planning process the first tier receives the demand plan from the Original Equipment Manufacturer (OEM) and manually computes its MRP. Then the MRP is exploded into the demand plans for each of its suppliers. The second tier receives the demand plan from the first tier and manually computes its MRP. The only exchange of information is the demand plan that the first tier sends to the second tier. Moreover, there is no collaboration in case the second tier cannot supply the required demand plan to the first tier. Therefore, the second tier has to incur in extra costs, such as inventory costs, urgent purchase costs, delay costs, etc., to meet the demand of components/materials of the first tier. MRPs are manually computed by the company planners, incurring in resources and capacity expenses due to the difficultness and time-consuming associated. The lack of affordable collaborative tools does not motivate enterprises to evolve from traditional to collaborative planning approaches, which involve the application of negotiation and communication mechanisms. The complexity of the collaborative planning process increases, due to the appearance of more restrictions defined by other partners, which sometimes could be conflicting and contradictory.

C2NET leverages the potential of Cloud technologies providing a manufacturing infrastructure for a real-time knowledge of different supply chain components such as manufacturing assets status, inventory levels or current demand at consumption points. By providing specific tools for optimisation and collaboration in the cloud, companies involved in a $\mathrm{CN}$ will be able to increase their enterprise resilience to respond quickly, flexibly and efficiently to changes in demand and unexpected events that have place during the planning process [7].

\section{Automated and collaborative calculation of the Materials Requirement Plan}

The three main modules of C2NET Architecture (C2NET DCF, C2NET OPT and C2NET COT OPP) are integrated in the C2NET Cloud Platform (C2NET CPL), in order to allow the industrial enterprises to automatically and collaboratively compute the MRP. A brief description of the components, embedded in the Cloud Architecture, 
involved in the automated and collaborative calculation process of the MRP is presented next:

Legacy Systems Hub: It virtualises legacy systems hub in order to upload to C2NET the data from the enterprise

C2NET UCP: It is a web-based application that provides user interfaces for companies users, for each C2NET solution.

C2NET DCF: It is a cloud-supported storage, in which the developments in open data contribute to the wide availability of such data. It also includes the mapping rules that allow transforming the companies' data into C2NET standardised and homogenised data.

C2NET OPT: To support the optimisation of manufacturing and logistics assets by the collaborative computation of production, replenishment and delivery plans. In C2NET OPT, the plan is characterised [8] according to the objectives, restrictions and the solving time. C2NET OPT contains a repository of algorithms that solve different set of individual or collaborative plans related to replenishment, production and delivery (including optimisation mathematical models, heuristic, metaheuristic and matheuristic algorithms). C2NET OPT is developed with the capability of selecting the most appropriate algorithm/s to automatically compute the defined plan taking into account its objectives, restrictions and solving time, considering the minimum GAP. The C2NET heuristics valid for the collaboration and automatically calculation of the MRP are [9]: (i) Lot-by-Lot, exactly provides what is needed, minimising the inventory costs. Both the periods interval between orders and the size of the batch are variable; (ii) Minimum Unit Cost, calculates the unit cost of requesting the net requirements of the 1 st period, calculating the $1^{\text {st }}+2^{\text {nd }}$ period, and so on until a relative minimum is reached; Indicator: unit cost $=$ (order cost + inventory cost)/units; (iii) Minimum Total Cost: Similar to the previous one but considering the total costs; and (iv) Silver-Meal, selects the batch that results in a minimum total cost (order cost + inventory cost) per period, for the interval covered by the replenishment; Indicator: cost per period $=($ order cost + inventory cost $) / \mathrm{n}^{\mathrm{o}}$ periods covered; (v) Minimum Period Cost (MPC): Similar to the previous one but considering the order and inventory cost per period $=($ order cost + inventory cost + extra purchase cost + urgent order cost of products $) / n^{0}$ periods covered.

C2NET COT OPP: provides a set of components for value-chain handling of collaborative manufacturing issues in each defined plan. It includes the following modules: (i) OPP Negotiation, defines the interaction between COT and the UCP modules during a workflow execution; (ii) OPP Notification, defines the interface used to notify events and actions related to the process of optimising plans affecting several companies in a network.

In order to compute the MRP the First Tier planner and the Second Tier planner register in the C2NET DCF all the sources of data from its legacy systems, (e.g. products, bill of materials, periods, demand plan, etc.). Some of the data can change along the time, for example the demand plan of the OEM, which can be updated every period and change from the previous demand plan. The data is stored in the C2NET DCF through using the interoperable rules and an ontology created in C2NET. Once all the data needed for calculating the MRP is stored, the automated collaborative replenishment (MRP) planning process starts with the release of the demand plan of OEM (DP ${ }^{\mathrm{OEM}}$ ), which is also stored in C2NET DCF. In this regard, the First and 
Second Tier planner define, through the C2NET UCP, the plans to be solved, in this particular case the plan type and subtype refers to Source_MRP (see [8]). Moreover, the objectives, restrictions and the solving time required to automatically compute the Source_MRP are also determined. The defined plans are stored in C2NET DCF.

C2NET OPT contains a repository of algorithms, and it is in charge of selecting the most appropriate algorithm to solve the defined plans, Source_MPR, according to the features assigned. The components assembled by the First Tier, are planned in the same period in which are served, synchronously with the OEM. While, components purchased by the First Tier, to the Second Tier, are computed by applying the MPC algorithm. MPC algorithm, allows the First Tier to accumulate orders for the purchased components, so that the global cost is minimised, balancing the order, purchase and inventory costs. The First Tier extracts from its MRP the Demand Plan of the Second Tier, which has a more discretized (grouped) demand. In this regard, the Second Tier could have problems on supplying the demand of some components in some periods. This is why the Second Tier calculates its MRP, using the MPC algorithm, and checks if it can really satisfy the discrete demand sent by the First Tier. In case the Second Tier cannot cope with the required demand, the Second Tier sends the First Tier the maximum amount of components that can supply. The First Tier computes again the MRP considering the restrictions sent by the Second Tier, with regards to the maximum amount of components that can supply, the First Tier computes again the MRP using the MPC algorithm. A negotiation loop is generated until the components demanded by the First Tier can be satisfied by the Second Tier. The MPC algorithm ensures not having a negative inventory, also allows to use, first the security stock, and when the security stock finishes, is when the MPC algorithm decides to buy the components incurring in a urgent purchase and extra order cost. The Holistic Algorithm for Source_MRP is described next:

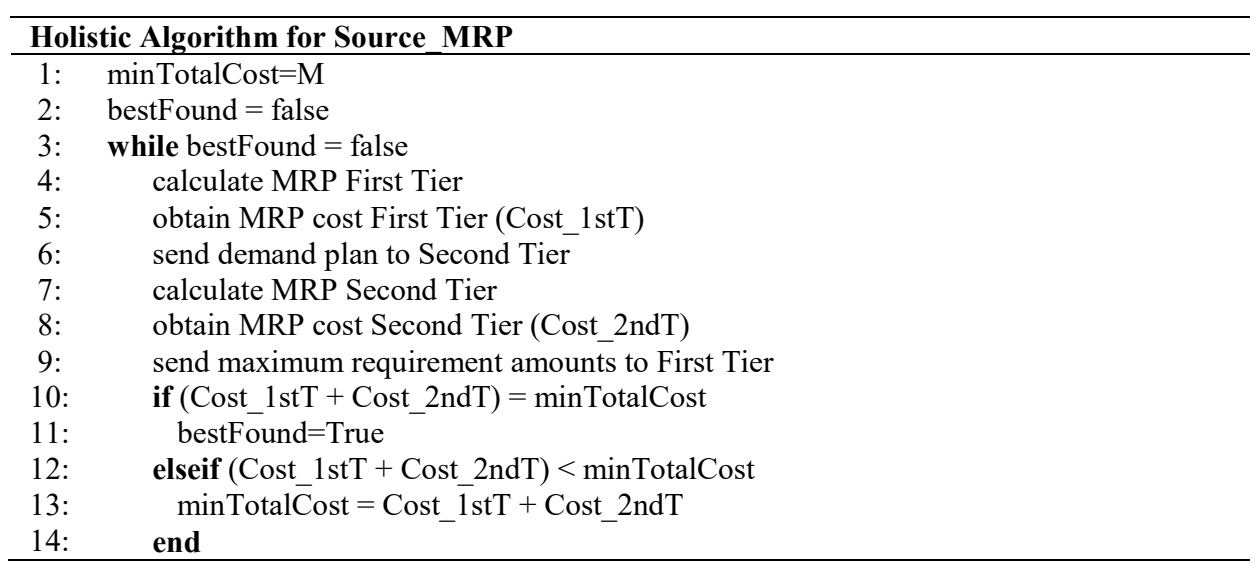




\section{Industrial Application in the Automotive Sector}

Cloud-supported storage collaboration and optimisation contributes to the wide availability of data, and afford enterprises to establish collaborative relationships and compute collaborative plans. In order to perform a collaborative MRP the C2NET DCF, C2NET OPT and C2NET COT are considered; allowing the First and Second Tier exchange new input data from the restrictions defined by the partners participating in the negotiation; this negotiation is draft in the workflow presented in Figure 2.

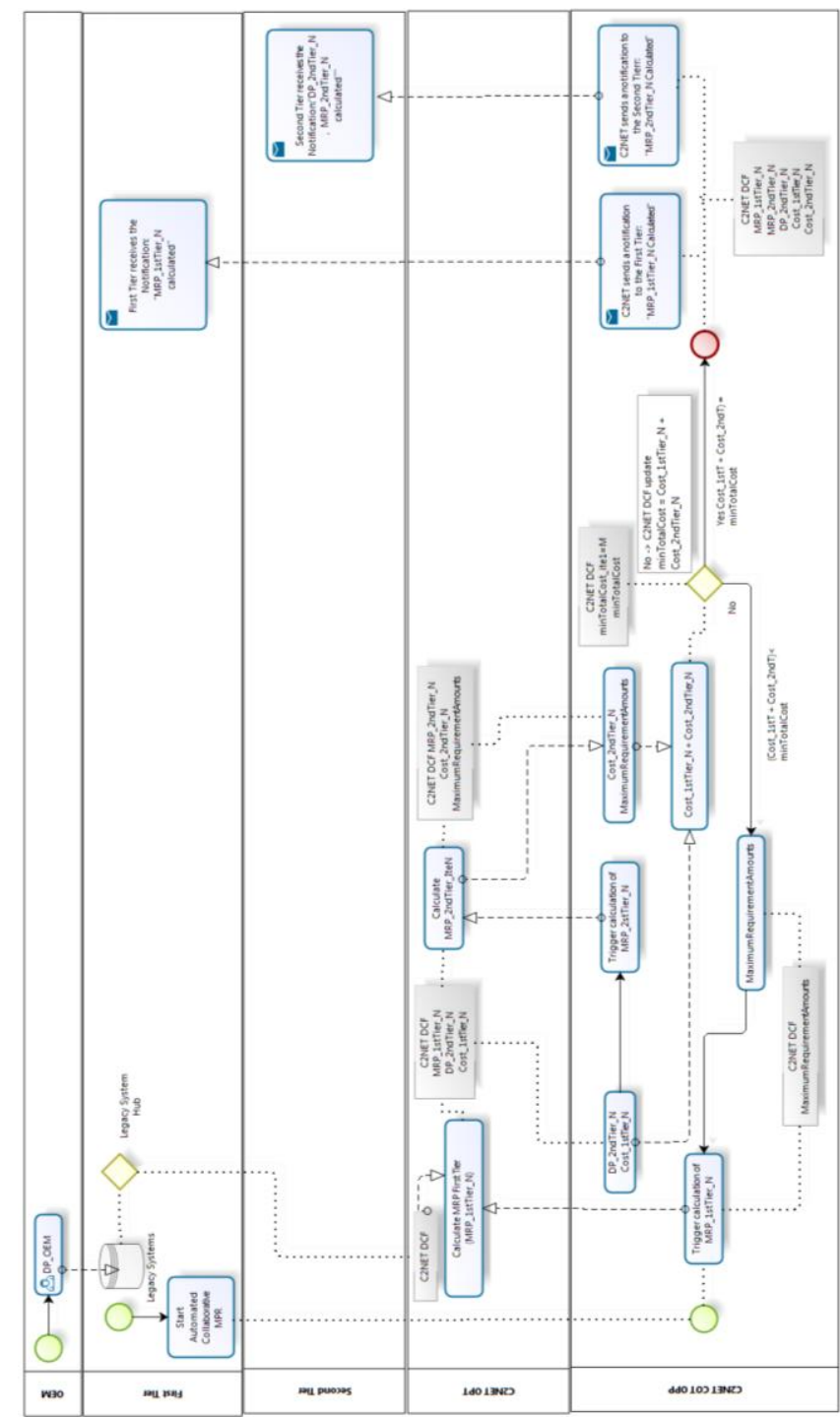

Fig. 2. C2NET COT OPP workflow designed for the collaborative automated MRP 
The data required to compute the collaborative MRP is stored in C2NET DCF. A database, with standardised structure, has been developed in C2NET project. C2NET database consists of Standardised Tables (STables) that contain all the information required to compute the MPC algorithm. The STables used are: (i) Part, contains information of the enterprise products; (ii) Period, contains information about the periods in which the MRP is computed, (iii) Part_Part, contains information of the BOM, (iv) Part_Period, contains information of the demand plans; and (v) Customer_Part, links the products of the First and Second Tier. For the developed example the First Tier works with 289 products, and the Second Tier works with 237 products. The MRP planning horizon is divided in 53 periods. Each network Tier has its own database and STables.

The holistic algorithm proposed, to automatically and collaboratively compute the Source_MRP plan, is validated through its application in a real scenario in the automotive sector; two network suppliers are considered, the First and Second Tiers. The collaboration negotiation loop initialises minTotalCost $t^{1}=M$. The First Tier receives the $\mathrm{DP}^{\mathrm{OEM}}$ and computes its own MRP, which has an associated cost (Cost_1stT). The demand plan of the Second Supplier is extracted from the First Tier MRP. The Second Tier computes its own MRP, according to the First Tier demand plan. If the Second Tier cannot completely satisfy the components demanded by the First Tier, sends the maximum amount of components that is able to supply; the rest of the components will be planned by considering an urgent purchase cost and an extra order cost. The resulting MPR has linked a cost (Cost_2ndT). The minTotalCost ${ }^{\mathrm{N}}$ is computed by the sum of the Cost_1stT and the Cost_2ndT. If this minTotalCost ${ }^{\mathrm{N}}$ is lower than the previous initialised, $\overline{\operatorname{minT} T o t a l C_{0} t^{1}}$, the new minTotalCost ${ }^{\mathrm{N}}$ is stored. The First Tier computes again the MRP by considering the new restrictions defined by the Second Tier, and a new cost is computed $\left(\right.$ Cost $\left.1 \mathrm{stT}^{\mathrm{N}}\right)$. The First Tier extracts the demand plan of the Second Tier and the negotiation process is repeated until a minTotalCost ${ }^{\mathrm{N}}$ is repeated (see Table 1). In Table 1, the first column is the number of the iteration; second column shows the TotalCost in each iteration; third column stores the minimum of the TotalCost obtained so far; fourth column is True when the TotalCost $=$ minTotalCost, False otherwise; fifth column is True if the minTotalCost has been computed in a previous iteration, False otherwise. The stopping rule of the negotiation process is defined when the minTotalCost is repeated. In the example, the $12^{\text {th }}$ iteration has the same minTotalCost as $6^{\text {th }}$ iteration and the stopping rule is accomplished, obtaining the result of the Fisrt and Second Tier Source_MRP. Looking at Table 1 , the TotalCost oscilate between two solutions (e.g. see Iterations $4,6,8,10$, and 12; and Iterations $3,5,7,9$, and 11), but it is not until the $12^{\text {th }}$ iteration when the minTotalCost is repeated, and the negotiation process stopped. 
Table 1. Iteration Results of the collaborative Source MRP

\begin{tabular}{rcccc}
\hline Iteration & TotalCost & minTotalCost & TotalCost $=$ minTotalCost & minTotalCost repeated \\
\hline 1 & 100,000 & 100,000 & True & False \\
2 & 91,260 & 91,260 & True & False \\
3 & 106,633 & 91,260 & False & False \\
4 & 89,527 & 89,527 & True & False \\
5 & 105,243 & 89,527 & False & False \\
6 & 89,398 & 89,398 & True & False \\
7 & 105,319 & 89,398 & False & False \\
8 & 89,402 & 89,398 & False & False \\
9 & 105,249 & 89,398 & False & False \\
10 & 89,527 & 89,398 & False & False \\
11 & 105,243 & 89,398 & False & False \\
12 & 89,398 & 89,398 & True & True \\
\hline
\end{tabular}

\section{Conclusions}

This paper is part of C2NET project results, proposing a novel holistic algorithm and data sharing to automatically compute the Materials Requirement Plan in a collaborative network, using the cloud environment. In general C2NET, and particularly the proposed holistic algorithm, allows dealing with the emerging challenges for establishing collaborative relationships. Previous approaches developed to address collaborative networking are constrained in terms of applied algorithms and mechanisms, to comply with this fast evolving scenario. C2NET proposes new solutions that consider convergent technologies, such as, IoT, Linked Data, Data Privacy, Big Data, etc. The proposed solution introduces an innovative algorithm aimed at fulfilling the lack of affordable tools for the collaborative planning, in the specific area of materials requirement plans. The reduction of costs associated to the collaboration highly improves the collaborative network stability and sustainability, fulfilling the vision of beneficial collaboration in current dynamic markets in which enterprises are embedded. Limitations associated with the application of the proposed holistic algorithm, for collaboratively compute the Materials Requirement Planning, is related with lack of agreements between the MRPs obtained results. Moreover, the proper application of the algorithm is influenced by the main drawback of collaborative networks, which are characterized by uncertainty and incomplete information, being a limitation the work of gathering all the data, required by the enterprises, in an accurate way to feed the holistic algorithm.

\section{Future Research}

The contribution has been verified and validated with two network partners of the automotive industry sector. Future work leads to design generic workflows considering different network typologies. C2NET project will start with a tree supply chain, typically observed in the automotive industry (see Figure 3). The negotiation 
workflow and proposed will be designed in order to be extensible in an automated way. The holistic algorithm used to compute the Source_MRP will take into consideration the restrictions given by all the network partners when negotiating. Moreover, negotiation rules will be specifically defined in each pair of nodes. The algorithms used, MPC, allows minimizing the normal and urgent order costs, the normal and extra purchase costs, and the inventory costs. In order compute the costs to the global network; new holistic algorithm will include procedures to redistribute and share costs in an equitable way, so that costs are assigned in a higher extent to the nodes that contribute to have extra purchases and urgent orders in the MRP.

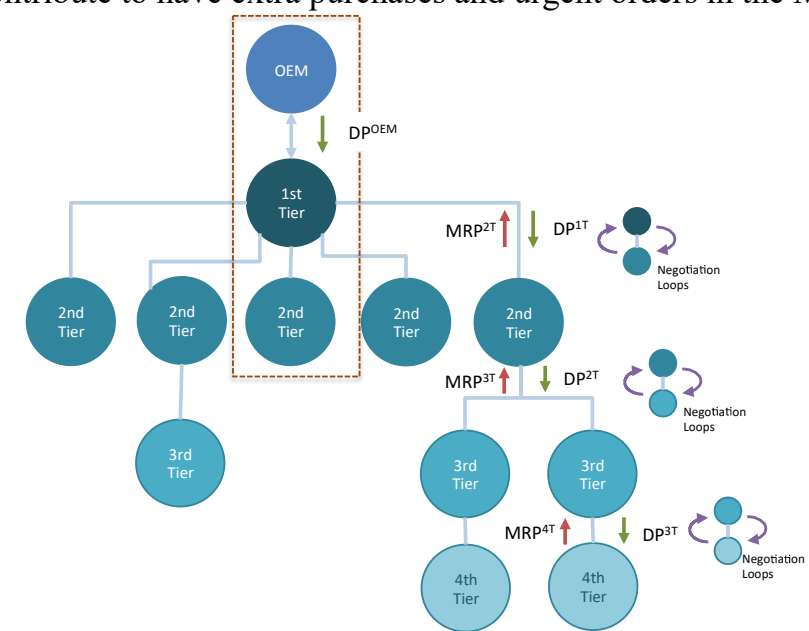

Fig. 3. Diagram for automated and collaborative calculation of the MRP in a tree collaborative network topology

Acknowledgments. The research leading to these results is in the frame of the "Cloud Collaborative Manufacturing Networks" (C2NET) project, which has received funding from the European Union's Horizon 2020 research and innovation programme under grant agreement No 636909

\section{References}

[1] CORDIS Europa, "Factories of the Future," H2020-EU.2.1.5.1. - Technologies for Factories of the Future, 2014. .

[2] H2020 Project C2NET, "http://cordis.europa.eu/project/rcn/193440_en.html," 2015. .

[3] B. Andres, R. Sanchis, and R. Poler, "A Cloud Platform to support Collaboration in Supply Networks,” Int. J. Prod. Manag. Eng., vol. 4, no. 1, pp. 5-13, 2016.

[4] B. Andres, R. Sanchis, J. Lamothe, L. Saari, and F. Hauser, "Integrated productiondistribution planning optimization models: A review in collaborative networks context," Int. J. Prod. Manag. Eng., vol. 5, no. 1, pp. 31-38, 2017.

[5] L. M. Camarinha-Matos and H. Afsarmanesh, "Collaborative networks : a new scientific discipline,” Journal of Intelligent Manufacturing, 16(4-5), pp. 439-452, 2005. 
[6] B. Andres and R. Poler, "Models, guidelines and tools for the integration of collaborative processes in non-hierarchical manufacturing networks : a review," Int. J. Comput. Integr. Manuf., vol. 2, no. 29, pp. 166-201, 2016.

[7] R. Sanchis, R. Poler, and F. C. Lario, "Identification and analysis of Disruptions: the first step to understand and measure Enterprise Resilience.," 6th Int. Conf. Ind. Eng. Ind. Manag., pp. 424-431, 2012.

[8] B. Andres, L. Saari, M. Lauras, and F. Eizaguirre, "Optimization Algorighms for Collaborative Manufacturing and Logistics Processes," in Enterprise Interoperability in the Digitized and Netwroked Factory of the Future, M. Zelm, G. Doumeingts, and J. P. Mendonça, Eds. iSTE, 2016, pp. 167-173.

[9] A. Orbegozo, B. Andres, J. Mula, M. Lauras, C. Monteiro, and M. Malheiro, "An overview of optimization models for integrated replenishment and producction planning decisions," in Building bridges between researchers and practitioners. Book of Abstracts of the International Joint Conference CIO-ICIEOM-IISE-AIM (IJC2016), 2016, p. 68. 